\title{
A Biologically Inspired Micro-Vehicle Capable of Aerial and Terrestrial Locomotion
}

\author{
Richard J. Bachmann, Frank J. Boria, Ravi Vaidyanathan*, Peter G. Ifju, and Roger D. Quinn
}

\begin{abstract}
The design, fabrication, and field testing of a $30.5 \mathrm{~cm}$ robot capable of aerial and terrestrial locomotion is described. The Micro Air-Land Vehicle (MALV) flies using a chord-wise, undercambered, bat-like compliant fixed wing and walks over rough terrain using passively compliant wheel-leg running gear. The vehicle successfully performs transitions from flight to walking and in some situations, from walking to flight. The lightweight ( 100g) carbon fiber vehicle flies, lands and crawls with a video sensor payload exceeding $20 \%$ its own mass.

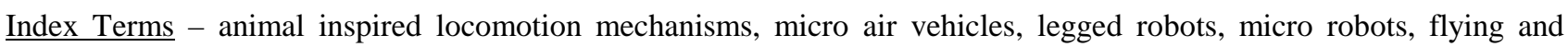
walking mobility
\end{abstract}

\section{INTRODUCTION}

A dvances in fabrication, sensors, electronics, and power storage have made possible the development of a wide range of small robotic vehicles capable of either aerial or terrestrial locomotion. Furthermore, insights into animal locomotion principles and mechanisms have significantly improved the mobility and stability of these vehicles. For example, the utility and importance of passively compliant wings for fixed-wing Micro Air Vehicles (MAVs) has been demonstrated for aircraft with wingspans as small as $10 \mathrm{~cm}$ [1]. Likewise, highly mobile ground vehicles using compliant legs have been constructed as short as $9 \mathrm{~cm}$ that can run rapidly over obstacles in excess of their own height [2].

This paper describes the design, fabrication, and testing of a novel small vehicle (dubbed the Micro Air-Land Vehicle (MALV)) that is capable of both aerial and terrestrial locomotion. Robot morphology is inspired by neuromechanics in animal locomotion, integrating passive compliance in both its wings, joints, and legs, such that it may fly, land, walk on the ground, climb over obstacles, and (in some circumstances) take to the air again all while transmitting sensor (video) feedback. At this time, experimental testing of the vehicle has been conducted in actual field conditions for the operations of surveillance, explosive detection, search/rescue, and remote inspection. Specifically, testing has shown MALV capable of deployment, flight, landing, crawling, and target inspection in a singe sequence of actions. The vehicle is capable of flying, crawling, climbing obstacles comparable to its height, and transitioning between locomotion modes. In the longer term, the design architecture and locomotion mechanisms are expected to lead to a family of vehicles of varying size, sensors, and range that may be configured for a wide range of applications.

Manuscript submitted October 10, 2007. This work was supported in part by the U.S. Department of Defense under Contracts FA8651-04C-0234 and FA8651-05HI-C-0097 and the NPS/US Special Forces Command (USSCOM) Cooperative Field Experimentation Program R. J. Bachmann is with BioRobots, LLC Cleveland, OH 44127 USA (r.j.bachmann@bio-robots.com).

F. J. Boria and P. G. Ifju are with the University of Florida, Gainesville, FL 32611 USA (boria@ufl.edu; ifju@ufl.edu).

Ravi Vaidyanathan* is with the University of Bristol, Bristol, UK, BS8 1TH (phone: +44 7970330 814, rxv@case.edu).

R. D. Quinn is with Case Western Reserve University, Cleveland, OH 44106 USA (dq@case.edu). 


\section{A. Overview and Design Approach}

In a biological organism, execution of a desired motion (e.g. locomotion) arises from the interaction of active (higherlevel) control centers with passive (lower-level) properties of the sensorimotor system, including the intrinsic mechanical response of the body. Animal "neuromechanical" systems successfully reject a range of disturbances that could otherwise induce instability or deformation of planned trajectories [43]. The first response to minimize such effects, in particular for higher frequency disturbances such as maintaining posture over varying terrestrial substrates and unexpected gusts in flight, is provided by the mechanical properties of the organism. In legged locomotion, for example, compliance (i.e. springs and dampers) plays a fundamental role in joints and structures that stabilize the body in an intrinsic fashion and thus greatly simplify higher-level control $[41,42]$. Reproduction of the dynamic properties of muscle and the intrinsic response of the entire mechanical system [44] has been a serious impediment to the successful realization of animal-like robot mobility over a variety of substrates and through different mediums (e.g. air and land). It is these intrinsic properties of the musculoskeletal system which augment neural stabilization of the body of an organism.

Although biological inspiration offers a wealth of promise for robot mobility, many constituent technologies are not at a state of maturity where they may be effectively implemented for small autonomous robots. Existing power, actuation, materials, and other robotic technologies have not developed to the point where animal-like neuromechanics may be directly integrated into robotic systems. Given this challenge, the majority of biologically inspired legged and flying robots have been confined to laboratory or limited field demonstrations. A method to surmount this, known as abstracted biological inspiration [8], focuses principally on the delivery of critical performance characteristics to the engineering system. Abstracted biological inspiration attempts to abstract salient biological principles and implement them using available technologies. This approach founded the basis of the design methodology aimed at delivering capabilities of flight locomotion, crawling locomotion, and transitions between the two to MALV.

\section{1) Organization of Paper}

The remainder of this section describes past work in flying and crawling micro robots respectively, in addition to some of the small body of research in robots with multimodal mobility. Section II delineates the biologically inspired structures for flight and walking used on MALV. Section III details design process and vehicle fabrication, while Section IV presents performance characteristics of the robot. Section V enumerates the conclusions of the research and envisioned future work.

\section{B. Micro Ground Vehicles}

Among the breadth of factors presently inhibiting the real-world deployment of terrestrial micro robots, we have noted two. First, the relative size of real-world obstacles (e.g. stairs, gravel, terrain fluctuations, etc.) makes movement difficult 
for small robots. For example, RHex ( $\sim 50 \mathrm{~cm}$ length), is the shortest robot to our knowledge that can climb standard stairs

[36]. Second, power-source miniaturization has not kept pace with other critical equipment such as actuation, sensing, and computation.

A wide array of vehicles has been constructed that attest to the difficulty of designing field-deployable terrestrial mobile micro-robots. Khepera robots have a $5 \mathrm{~cm}$ wheelbase, onboard power, and an array of sensors [3]. Although they are widely used by group behavior researchers, their $1.4 \mathrm{~cm}$ diameter wheels restrict them to operation on very smooth, flat surfaces. Millibots [4] use tracks, but it is not clear that they offer significant advantages since it is difficult to implement a modern track suspension at this small scale. A small hexapod has been developed by Fukui et al. [5] which runs in a tripod gait using piezoelectric actuators. However, small joint excursions also limit the vehicle to relatively flat surfaces. Birch et al. [6] developed a $7.5 \mathrm{~cm}$ long hexapod inspired by the cricket and actuated by McKibben artificial muscles. Though capable of waking using 2 bars of air pressure it has not yet carried its own power supply. Sprawlita [7] is a 16cm long hexapod which uses a combination of servomotors and air cylinders. Sprawlita attains a top speed of 4.5 body lengths per second, which is fast compared to existing robots of similar size. However, an operating air pressure of 6 bars makes it unlikely that the robot will become autonomous in its current form.

Abstracted biological inspiration has spawned a group of highly mobile robots, called Whegs ${ }^{\mathrm{TM}}$ [35] and Mini-Whegs ${ }^{\mathrm{TM}}$ [37]. At present, Mini-Whegs ${ }^{\mathrm{TM}}$ is one of the fastest small terrestrial vehicles that is also capable of surmounting large obstacles relative to its size. Using a single drive motor, the $9 \mathrm{~cm}$ long robot has run at 10 body lengths per second, and can easily run over $3.5 \mathrm{~cm}$ tall obstacles - higher than its body. The more recently developed iSprawl [32] also uses single motor propulsion and benefits from abstracted biological principles. It has run even faster, 15 body lengths per second, although its obstacle climbing ability is more restricted because of the relatively small range of motion of its feet.

\section{Micro Air Vehicles (MAVs)}

The majority of research to develop practical non-rotating winged MAVs can be broadly categorized into three fundamental approaches. The first and most widely used is to configure the airframe as a lifting body or flying wing using propeller-driven thrust in a manner similar to larger aircraft. With this approach, the emphasis is to increase the relative area of the lifting surface while decreasing drag, directly addressing the decrease in the aerodynamic efficiency, and putting less emphasis on of stability and control. Research groups have used optimized rigid wings and accepted the need for stability augmentation systems or superior pilot skill to address intrinsically unsteady behavior. Among the most successful examples of rigid wing MAVs designed with this approach is Aerovironment’s “Black Widow” [13], a 15cm flying wing. Virtually every component on the aircraft is custom built, including a sophisticated gyro-assisted control system. Other 
successful examples of this approach include the "Trochoid" [14] and the "Microstar MAV" [15]. Both of these also rely heavily on gyro-assisted stabilization systems for controllable flight. This approach differs significantly from natural flight: Birds and bats have well-defined wings and a fuselage; in nature we find no examples of lifting bodies or flying wings.

The second approach that is being explored for MAV design draws on direct biological inspiration through mimicry of insect or bird-like beating wings [9]-[12]. Flapping wings can produce both lift and thrust. Researchers have demonstrated flapping wing MAVs that can fly and even hover [45] using the "clap and fling” mechanism as described by Ellington [9]. However, these MAVs are susceptible to failure in even light winds and their payload capacity is very small. This approach remains attractive for future work, in particular for low speed, low wind applications such as inside buildings.

In a third approach [16]-[22], the lifting surface is allowed to move and deform passively like animal wings, which leads to more favorable aerodynamic performance in a fluctuating low Reynolds number environment. These findings helped lead to a flexible wing concept, which has been applied by Ifju to successful MAVs over the past eight years [1],[23]-[25]. Based upon this abstracted biologically inspired mechanism, flight vehicles have been developed that use conventional propeller-driven thrust in combination with an adaptive-shape, compliant wing that responds to flight conditions, and also develops a stable limit-cycle oscillation during flight.

\section{Multi-mode Mobility}

While the aerial and terrestrial vehicles described above represent significant achievements in their respective fields, their utility is limited by their inherent design optimization for a single locomotion modality. At present, very few robots have been developed that are capable of multiple modes of locomotion, with the majority of work focusing on swimming/crawling robots. One example is Boxybot [26], which uses a vertically oriented tail, and two horizontally oriented fins for aquatic propulsion. By reversing the orientation of one or both of the fins, turning moments or reverse thrust can be generated. Continuous rotation of the fins produces a kind of terrestrial movement. A water tight version of RHex [27] has also been equipped with fin-like legs that allow it to swim under water. The neuromechanical design of a more recent amphibious robot is based upon salamanders and it can run on land and swim using the same central pattern generator [48]. To the knowledge of the authors, the only other published work with the stated goal of both aerial and terrestrial locomotion is the Entomopter [28], being developed at Georgia Tech Research Institute. The Entomopter uses Reciprocating Chemical Muscle [29] to produce flapping motion of its four wings. We are not aware of data on the vehicle’s terrestrial capabilities or performance in both locomotion modes.

Nature has repeatedly demonstrated the need for multiple modes of locomotion, especially for small animals. Pure terrestrial locomotion may be impractical at this scale simply because of the distances that must be traveled to search for 
food, mates, etc. However, mono-modal aerial locomotion is also undesirable because it is impossible to stay airborne indefinitely. A variety of conditions (winds, etc.) make it difficult to land at an exact location, and walking is far more energy efficient than flying over short distances.

Utility for small robots often reflects the exact same problem domain as small animals; multiple modes of locomotion would represent a generational leap in their capability. Flight capacity could allow a vehicle to travel long distances and approach a general target area, while crawling locomotion would allow a range of additional possibilities (close inspection, surveillance, performance of tasks, etc.) unachievable by any vehicle existing today.

\section{BIOLOGICALLY INSPIRED STRUCTURES FOR FLYING AND WALKING}

As stated earlier, abstracted biological inspiration focused on functionality of the MALV with technology presently available. Its mechanical design incorporated neuromechanical flight (deformable wing) and walking (compliant wheellegs and axle joints) mechanisms, which were key to MALV’s locomotion capacity. The challenge was designing mechanisms to enable functionality in both modes while preserving as much overall mobility as possible in each individually.

\section{A. Terrestrial Locomotion}

The implementation of biological locomotion principles holds considerable promise for terrestrial locomotion. Legged animals exist and thrive at a wide range of sizes, and are capable of overcoming obstacles that are on the order of their own size. Animal legs behave as if they have passive spring-like compliant elements when they are perturbed [41]. Alexander describes three uses for springs in legged locomotion, including energy absorption [40]. Jindrich and Full demonstrated this in an experiment wherein a cockroach was suddenly perturbed, too quickly for its nervous system to react [42]. It was shown that the passive compliance in its legs stabilized its body. Similar stability benefits are achieved through compliant elements in the legs of the RHex robot [36]. Locomotion studies on cockroaches have also elucidated several critical behaviors that endow the insect with its remarkable mobility [34]. During normal walking, the animal uses a tripod gait, where adjacent legs are $180^{\circ}$ out of phase. The cockroach typically raises it front legs high in front of its body, allowing it to overcome smaller obstacles in stride, but when climbing larger obstacles, the animal moves adjacent legs into phase, thus increasing stability.

These benefits have been captured in design through the Whegs ${ }^{\mathrm{TM}}$ concept (Fig. 1), which has spawned a line of robots that implement abstracted biological inspiration (based on the cockroach) for advanced mobility. Compliance is implemented into Whegs ${ }^{\mathrm{TM}}$ legs in two ways, radially for shock absorption and torsionally for gait adaptation [8]. Torsional compliance allows for a single motor to drive six three-spoke wheel-leg appendages to accomplish all of the locomotion 
principles discussed above. Whegs ${ }^{\mathrm{TM}}$ robots are also scalable, with successful robots being developed with body lengths ranging from $89 \mathrm{~cm}$ down to $9 \mathrm{~cm}$.

This concept has been extended to Mini-Whegs ${ }^{\mathrm{TM}}$ (Fig. 2) that offer a combination of speed, mobility, durability, autonomy, and payload for terrestrial micro robots. Mini-Whegs ${ }^{\mathrm{TM}}$ robots are extremely fast (10 body-lengths per second) in comparison to most other legged robots, and can climb obstacles taller than the top of their bodies [36][37]. The wheelleg appendage results in a natural "high stepping” behavior, allowing the robot to surmount relatively large obstacles. These vehicles have tumbled down concrete stairs and been dropped from heights of over 10 body lengths, without damage. MiniWhegs ${ }^{\mathrm{TM}}$ have also carried over twice their body weight in payload [37].
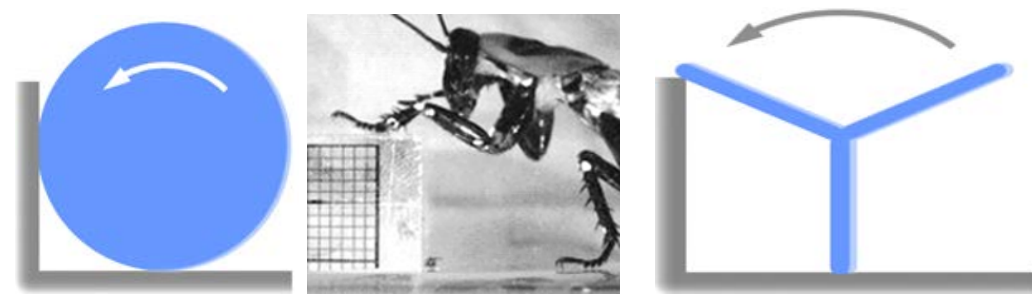

Fig. 1. The wheel-leg compromise between efficiency and ease of propulsion of a wheel and terrain mobility of legs.

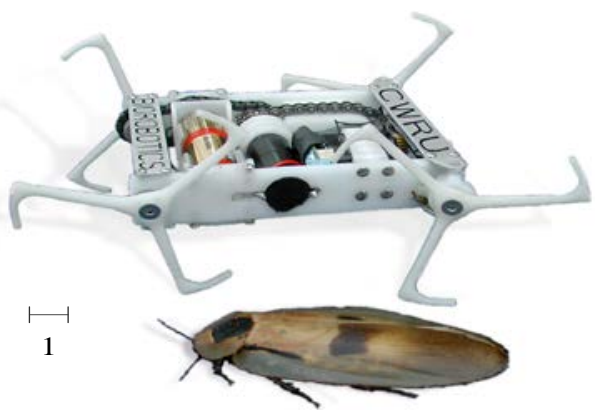

Fig. 2. Photograph depicting the relative sizes of a Mini-Whegs ${ }^{\mathrm{TM}}$ and a Blaberus giganteus cockroach

\section{A. Compliant wings for Aerial Locomotion}

A rigid leading edge, chord-wise compliant wing design is the basis for MALV's aerial locomotion. The compliant wing inspired by flying animals has several advantages over similarly sized rigid-wing vehicles [1]. Delayed stall allows the vehicles to operate at slower speeds. Improved aerodynamic efficiency reduces the payload that must be dedicated to energy storage. Passive accommodation to gust significantly improves stability.

It has been well established that the aerodynamic efficiency of conventional (smooth, rigid) airfoils is significantly compromised in the Reynolds number (Re) range between $10^{4}$ and $10^{6}$. This Re range corresponds to the class of craft referred to as micro air vehicles [38]. In fact, the ratio of coefficient of lift $\left(C_{L}\right)$ to coefficient of drag $\left(C_{D}\right)$ drops by nearly two orders of magnitude through this range. With smooth, rigid wings in this Re range, the laminar flow that prevails is easily separated, creating large separation bubbles, especially at higher angles of attack [39]. Flow separation leads to sudden increases in drag and loss of efficiency. The effects of the relationship discussed above are very clear in nature. Consider, for example, the behaviors of birds of various sizes. Birds with large wingspan, with a fixed wing $\operatorname{Re}>10^{6}$, tend to soar for prolonged periods of time. Medium-sized birds use a combination of flapping and gliding, while the smallest birds, with a fixed wing $\operatorname{Re}<10^{4}$, flap continuously and rapidly to stay aloft [18].

Other major obstacles exist for flight at this scale [1]. Earth’s atmosphere naturally exhibits turbulence with speeds on 
the same scale as the flight speed of MAVs. This can result in significant variations in airspeed from one wing to the other, which in turn leads to unwanted rolling and erratic flight. The small mass moments of inertia of these aircraft also adversely affect the stability and control characteristics of the vehicles. Even minor rolling or pitching moments can result in rapid movements that are difficult to counteract.

A rigid leading edge, chord-wise compliant wing addresses these issues for MALV's aerial locomotion capabilities. Through the mechanism of passive adaptive washout, a chord-wise compliant wing (first implemented at the University of Florida (UF) [1]) overcomes many of the difficulties associated with flight on the micro air vehicle scale. Adaptive washout is a behavior of the wing that involves the shape of the wing passively changing to adapt to variations in airflow. For example, an airborne vehicle may encounter a turbulent headwind, such that the airspeed over only the right wing is suddenly increased. The compliant wing structure responds to the instantaneous lift generated by the gust to deform in a manner similar to Fig. 3. This is called adaptive washout, and results in a reduction in the apparent angle of attack, and a subsequent decrease in lifting efficiency, as compared to the left wing. However, because the air velocity over the right wing is higher, it continues to develop a nearly equivalent lifting force as the left wing. Similarly, as the airflow over the wing stabilizes, the wing returns to its original shape. This behavior results in a vehicle that exhibits exceptionally smooth flight, even in gusty conditions (our own MALV flight tests have been conducted in the presence of winds that precluded the flight of larger (2 meter wingspan) rigid aircraft.

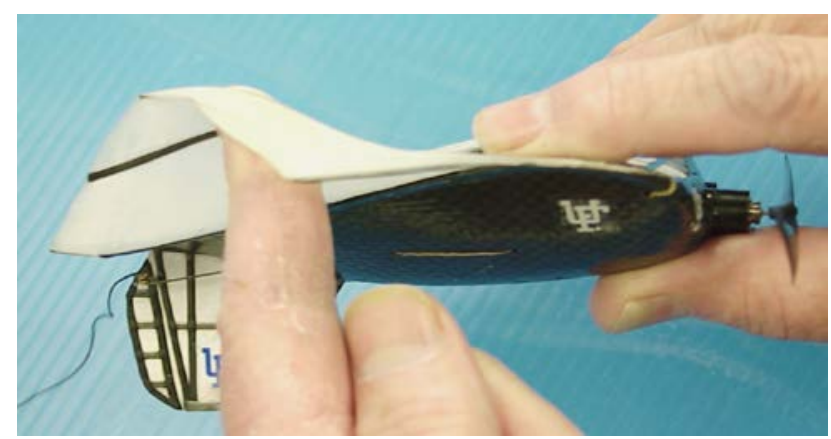

Fig. 3. The chord-wise compliance of a flexible wing allows for passive adaptive washout, increasing aircraft stability.

\section{MALV DESIGN AND DEVELOPMENT}

\section{A. Design Methodology}

\section{1) Locomotion Mechanisms}

The function of MALV is to carry a sensor payload, fly a long distance, and then land and move on the ground for a short distance, all while relaying visual information to a home base. For flight, a flexible wing was first selected over rigid or flapping wings to provide the best combination of controllability and payload capacity with the critical size range. Next, 
we considered terrestrial locomotion mechanisms that could be integrated into a MAV. One possibility was to attach freespinning wheels to the fuselage of a MAV and use its propeller to drive the vehicle on the ground and in the air. Our experiments demonstrated that such a vehicle can land and take-off from smooth, firm terrain. However, this device had extremely poor ground mobility on rugged terrain. The propeller had a strong tendency to collide with obstacles, thus severely restricting ground mobility. Ground locomotion also suffered; the forward thrust of the propeller was, out of necessity, above the wheel axle, creating a torque that pitched the vehicle forward. When the wheels contacted an obstacle, the vehicle would often pitch forward nose first rather than actually moving forward. A possible alternative to this involved directly powering wheels attached to the fuselage, yet the vehicle's mobility would still be limited as it would not be able to climb obstacles even a small fraction of its own height. Finally, legged locomotion mechanisms were judged to be too complicated, delicate, and heavy at this time for use on a vehicle capable of both flight and crawling. We therefore chose to integrate flexible-wing and wheel-leg (Whegs ${ }^{\mathrm{TM}}$ ) mechanisms to design MALV.

\section{2) Multi-Modal Mobility Tradeoffs}

A design analysis was performed to determine how best to integrate flight and ground mobility mechanisms. If a MiniWhegs ${ }^{\mathrm{TM}}$ crawling robot was simply attached to the bottom of a MAV, the resulting vehicle would be too heavy to fly unless its wingspan was greatly increased. Therefore, a tradeoff analysis was done to determine the most important parameters for ground locomotion and MAV locomotion. The successful MALV design preserves those parameters as much as possible. Less important design parameters were compromised to achieve the best possible vehicle performance. In the case of a conflict between parameters important for flight versus ground mobility, a morphing mechanism was employed to resolve the problem. In the tradeoff analysis, flight was assumed to be the limiting condition because of energy demands and the larger payload enabled by crawling structures.

In flight, legs increase drag and reduce controllability. Furthermore, their associated mass reduces payload and can alter flight stability. On the ground, wings, propellers, and tails limit payload and impede mobility in confined spaces. The fuselage of an aerial vehicle tends to be long to increase its stability, but on the ground a long chassis causes the vehicle to high center on obstacles. On the ground, more legs can increase a vehicle’s stability and mobility, but in the air they add drag and mass. These design inconsistencies broadly fell into two categories: mass and geometry.

Wheel-legs were judged vital to the ground mobility of MALV. However, their implementation was reconsidered to improve the overall performance of the vehicle. Past wheel-leg robots in small sizes typically had four wheel-legs driven by one propulsion motor and its front wheel-legs are steered. The front wheel-legs are most important because they reach in front of the vehicle and on top of obstacles in the vehicle's path to lift and pull the vehicle forward. Whegs ${ }^{\mathrm{TM}}$ are designed this way to model the front feet of cockroach, which lift high and in front of the animal to overcome obstacles [34]. To 
reduce mass and complexity, we tested the ground mobility of a MALV with two wheel-legs instead of four. The wheellegs were placed in the front and to the side of the propeller. The rear of the fuselage dragged on the ground. We found that a MALV with this configuration could move forward over obstacles similar in height to a comparably sized Mini-Whegs ${ }^{\mathrm{TM}}$ robot. Additionally, the fuselage provided a tail-like action that prevented the robot from flipping onto its back, which happens when a purely terrestrial vehicle attempts to surmount obstacles that are taller than its own height. The drawback to this design is that MALV's mobility in reverse on rugged terrain was poor because the fuselage impacts surface irregularities and impedes motion. However, the weight savings justified the two-wheel leg design.

Wheel-leg steering versus differential steering was also contrasted in tradeoff design studies. Wheel-leg steering requires the wheel-legs to be placed further outboard on the wings so they do not strike the propeller when they are turned. Either design requires two motors. The differential steering design was chosen because no steering mechanism is required; the design is simpler and enables a much smaller turning radius.

Efficient hybrid designs can reduce mass by integrating structural, sensor, actuator and power components. In MALV, the fuselage of the aircraft is also the chassis of the ground vehicle. Its shape has been changed to meet design criteria for flight and ground systems. MALV uses the same cameras in flight and ground locomotion to transmit video to a remote base. The same motor could be used for both flight and ground mobility in a manner akin to insects using large muscles to drive their body-coxa leg joints and flap their wings [46, 47]. However, this idea was abandoned for the first-generation robot because the complexity overrode possible mass reduction. A transmission would be needed because the wheel-legs must turn much more slowly than the propeller. The propeller shaft and wheel-leg axles are perpendicular, which also increases transmission complexity. Furthermore, a clutch would be needed to switch from propeller to wheel-leg drive. For these reasons we chose to use different motors for flight and ground mobility.

Wings provide a geometric inconsistency that can not be compromised. Wings are clearly essential for flight, but are an impediment for ground locomotion especially when MALV is moving through narrow spaces. Birds and insects fold their wings when they are on the ground to eliminate impediments to motion. An insect-like wing folding mechanism has been developed for MALV that mimics this action. It can stow its wings on its back to move more readily and without restriction on the ground.

\section{3) Design Summary}

Simply attaching wheel-legs to a MAV was inadequate to design an efficient MALV. As is described above and in more detail in the following, we optimized a range of parameters for tradeoff in MALV's design space, compromised where necessary, and implemented a systems approach focused on coupling between mechanisms for the design of each component. The resulting MALV achieves its goals of air and ground locomotion, but because of necessary design 
tradeoffs it is not yet as agile on the ground as Mini-Whegs ${ }^{\mathrm{TM}}$ and has less payload capacity and is less controllable than a flexible-wing MAV.

\section{B. MALV's Design}

Based upon weight estimates and initial flight testing, the lift capacity of a $30 \mathrm{~cm}$ wingspan MAV was determined to be sufficient to carry the additional weight associated with components needed for terrestrial locomotion ${ }^{1}$. Analysis of existing technology led to the selection of R/C hobby servos to drive the wheel-leg appendages. Modified R/C servos were chosen due to their low weight and ease of implementation. The modification allows for continuous servo rotation after which the integrated position control electronics act as speed controllers so that the servos can receive commands and power directly from the receiver. This process avoided the weight of additional speed controllers. Since one R/C servo was used for each wheel-leg, this configuration required a five channel receiver (flight motor, elevator, rudder, left wheel-leg motor, and right wheel-leg motor), subsequently adding mass. The extra mass associated with the implementation of terrestrial walking was approximately $25 \mathrm{~g}$, resulting in a $120 \mathrm{~g}$ projected mass.

Beginning with the estimated total vehicle mass of $120 \mathrm{~g}$ and the established maximum dimension of 30 centimeters, a new compliant wing was designed to provide the necessary flight characteristics for the vehicle. A software package (designed at the University of Florida) was used to identify an efficient wing shape, within the defined parameters, capable of producing the necessary lift. Once satisfactory wing design parameters are identified, an output script file is generated. This script file is then imported into a CAD program (Fig. 4), where it is converted into CNC tool paths for milling of a wing mold upon which the wing will be laid out during composite fabrication. The software automatically scales and translates the airfoil shape so that the entire leading edge of the wing lies in a horizontal plane.

After the wing tool is milled, it is prepared for the fabrication process [31]. First, a layer of release film is applied to the tool to prevent any resins or adhesives from bonding to and damaging the tool. A schematic of the wing layout (including the leading edge, battens, and canopy) is placed on the tool, and a second layer of release film is applied. Using unidirectional and woven resin-impregnated (prepreg) carbon fiber, the wing structure is laid out on the tool. The wing fabric, a polycarbonate coated polypropylene, was selected due to its compliant properties akin to a natural wing. The fabric is then overlaid onto the structure. Additional layers of carbon fiber were applied to form a skeleton for the wing akin to a flying animal's wing. The carbon fiber skeleton maintained the general shape of the wing while still enabling the polypropylene to warp in flight for neuromechanical stability. Samples of wing tools with an array of completed wings and tail sections are pictured in Fig. 5. It should be noted that the compliant wing and skeletal structure may also enable MALV

\footnotetext{
${ }^{1}$ Initial performance specifications also called for a wingspan $<33 \mathrm{~cm}$ for device portability.
} 
to fold its wings in an insect-like manner (Fig. 6).

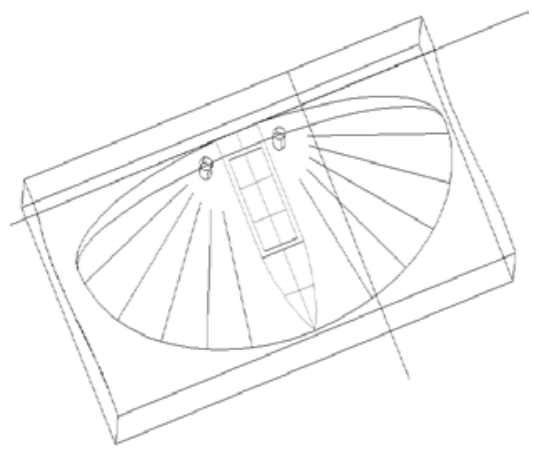

Fig. 4. A CAD solid model of the wing tool

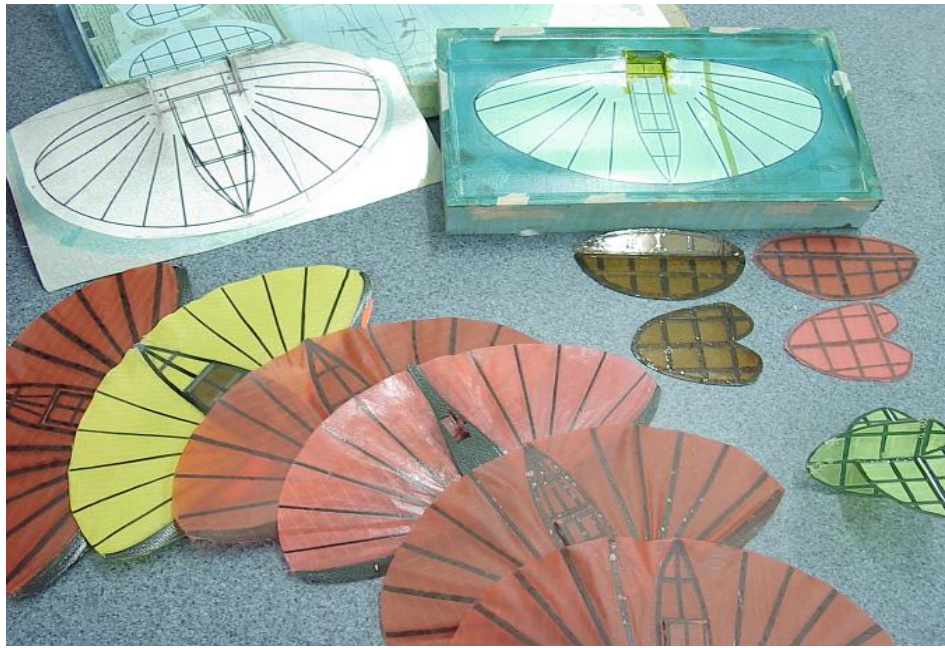

Fig. 5. Wing tools, tail sections, and fabricated wings.

In addition to wing selection, the fuselage had to be designed to integrate terrestrial walking. Three issues were of critical importance in MALV fuselage design: 1) for durability - physical incorporation of the wheel-leg drive motors within the fuselage, 2) for flight and land mobility - maintaining the desired horizontal position of the center of gravity (CG), and 3) for obstacle climbing - locating the wheel-legs forward such that their feet contact obstacles before any other part of the vehicle. This wing analysis identified the theoretical location of the wing aerodynamic center (AC). Pitch stability of an aerial vehicle is maintained by locating the CG forward of the AC. If the down-force generated by the tailsection is too large, the vehicle pitches up, resulting in a more positive angle of attack for both the wing and the elevator. The changes in lift from the two surfaces counteract the original discrepancy between the desired and actual moment balance on the vehicle. Fortunately, the second and third considerations are complementary because placing the wheel-legs forward on the fuselage also moved the CG forward.

After assembling a list of the components and their masses, the fuselage length was determined to accommodate placement of the CG in the desired horizontal location with the wheel-legs in the front of the vehicle. The fuselage was widened for the wheel-leg drive motors. Fuselage tools, array of wing fabrics, and completed MALV vehicles are shown in Fig. 7.

To take full advantage of the strength and weight of the carbon composite material, tools were fabricated to integrate the servomotor foot into the wheel-leg (Fig. 7). The carbon fiber also serves to reinforce the nylon servo horn in this configuration. The tool allows for endowing the wheel-leg spokes with the desired "splayed" shape. This shape allows the wheel-legs to reach out around (and in front of) the propeller (vehicle in lower right corner of Fig. 6), while minimizing the necessary width of the fuselage. 


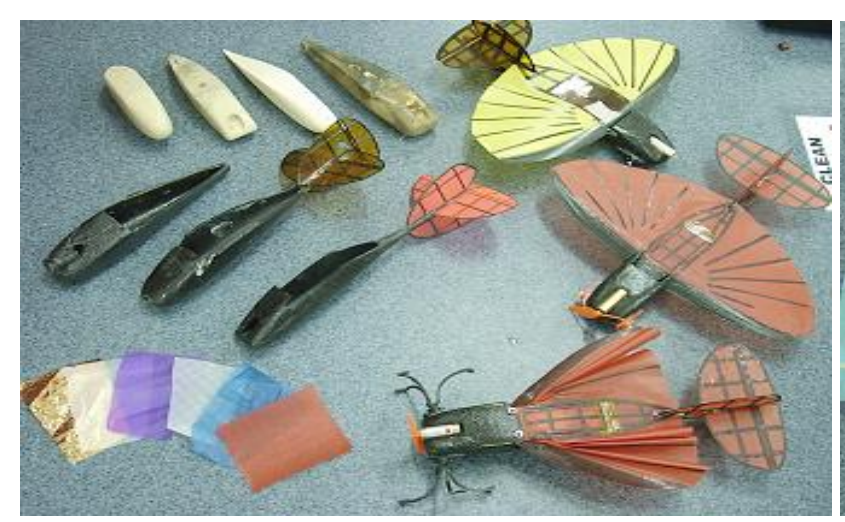

Fig. 6. Three completed vehicles, a series of fuselage tools, and an array of investigated wing fabrics. The bottom right vehicle shows a wing folding concept for future work.

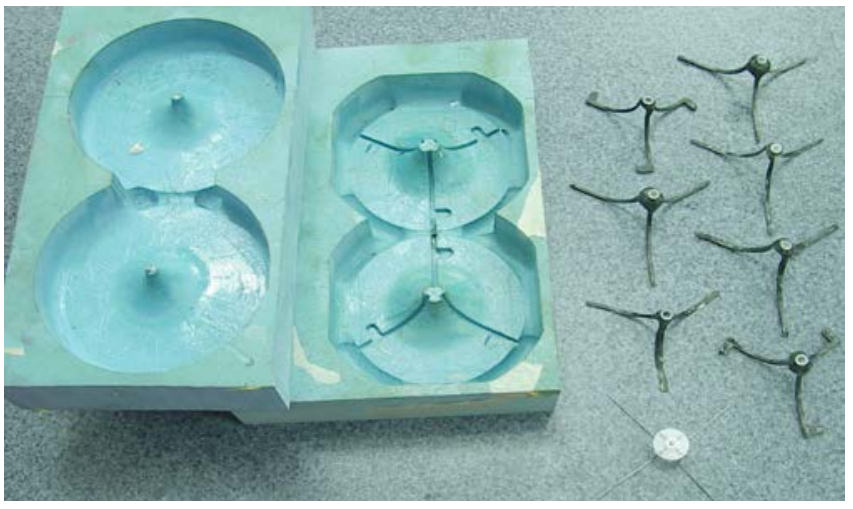

Fig. 7. Two wheel-leg tools and several tested designs

In addition to enabling mobility, compliant structures in biological organisms also help to reduce damage to mechanical elements during impact. It is of critical importance to MALV to maintain the functionality of the vehicle during the impact of landing. Multiple wheel leg designs for MALV were evaluated against two criteria: 1) surviving the landing process, and 2) facilitating terrestrial locomotion. During landing, a large torsional impact load is placed on the entire system, in particular terrestrial locomotion components including the wheellegs and the drive motors. Traditionally, robot design has striven to maximize the impedance between actuator and load and to minimize joint compliance, given that compliance can introduce uncontrollable and underactuated degrees of freedom. For MALV, advantages of leg or joint compliance ideally will lead to: 1) lower inertial forces with compliant joints, 2) lower reflected impedance with drive motors, 3) potential for efficient (elastic) energy storage and restitution, 4) passive dynamical compensation for destabilizing effects resulting from transmission lag, and, most importantly for landing and crawling in sequence, and 5) greater shock tolerance and reduced damage due to the "low-pass filter" (neuromechanical) properties of compliant/elastic elements.

It was found that compliant wheel-legs and axles lent dynamic mechanical properties enabling these advantages. Testing demonstrated these were durable, could survive landing impact, and to best protect the system (drive motors, etc.) by providing neuromechanical rejection of high frequency, high impact disturbances. The simplest design implemented was a four-spoke wheel-leg fabricated from spring tempered stainless steel wire (shown at the bottom of Fig. 7). A wire diameter of $1.19 \mathrm{~mm}$ (0.047 inches) provided sufficient compliance to absorb the landing impact, but not buckle undesirably during terrestrial locomotion. An improved design used the compliance of the spring steel, implementing an efficient foot shape (identified in previous crawling research [33]), and incorporating joint compliance in the axle to provide much improved performance. Fig. 8 shows the 
resulting wheel-leg. The closed loop foot avoids difficulties associated with sharp spokes becoming stuck in the substrate, compliance in the steel and leg shape increases mobility, and compliance in the axle and leg insulates the terrestrial crawling system from damage on harsh impact.
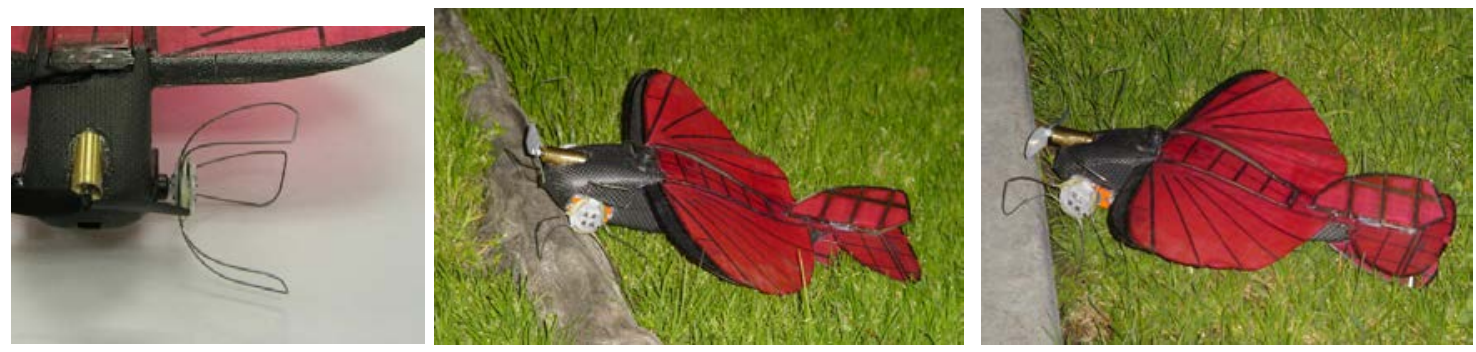

Fig. 8. MALV II has piano wire wheel-legs that are compliant on landing and resist becoming embedded in the substrate. These wheel-legs also enable it to crawl through grassy areas and climb obstacles taller than its leg length.

\section{RESUlts AND PERFORMANCE TESTING}

\section{A. Vehicle Description}

Several iterations of the design and fabrication process led to the completion of a range of vehicles capable of aerial and terrestrial locomotion. The first vehicle had a solid carbon fiber wing which was built principally for testing the integration of wheel-legs with basic flying mechanisms. While significantly limiting the flexibility of the wing surface, this arrangement did allow for the implementation of aileron control, which provides more responsive control than the rudder/elevator system implemented on the second generation vehicle (MALV II). However, the increased passive stability incorporating the bio-inspired chord-wise compliant wing led to the adoption of MALV II as the standard vehicle. Table I lists the physical characteristics of MALV II.

\begin{tabular}{|lc|}
\hline Parameter & Value \\
\hline Overall length & $30.5 \mathrm{~cm}$ \\
\hline Mass & $118 \mathrm{~g}$ \\
\hline Fuselage length & $21.6 \mathrm{~cm}$ \\
\hline Fuselage width & $5.1 \mathrm{~cm}$ \\
\hline Location of CG from fuselage tip & $9.5 \mathrm{~cm}$ \\
\hline Location of leading edge & $6.9 \mathrm{~cm}$ \\
\hline Leg length & $4.2 \mathrm{~cm}$ \\
\hline Track (distance between wheel legs) & $16.7 \mathrm{~cm}$ \\
\hline
\end{tabular}

Table I. Physical characteristics of MALV II. The location of the CG and the location of the wing leading edge are along the longitudinal axis, measured from the tip of the fuselage.

The design process was predicated on the knowledge of the masses of the components that would be included in the vehicle. Components were selected to provide the necessary thrust, control, sensing and terrestrial power 
to perform the desired tasks. Table II enumerates the critical internal components of the vehicle.

Iterations on the MAVLab software generated the wing with the highest efficiency $\left(C_{L} / C_{D}\right)$, within the prescribed dimensions that could generate the required lift at $9 \mathrm{~m} / \mathrm{s}$ airspeed. This value is derived from prior experience piloting flexible wing MAVs. Beyond this speed, the vehicle becomes more difficult to pilot. Wing parameters are listed in Table III.

The wing planform is bounded by semi-ellipses at the leading and trailing edges. The root chord length of the wing is $15 \mathrm{~cm}$, with the maximum width occurring $4.75 \mathrm{~cm}$ from the leading edge. The wing was mounted to the fuselage with an incidence angle of $8^{\circ}$, with respect to the thrust line of the motor.

Control surface parameters are shown in Table IV. The horizontal stabilizer was mounted parallel to the motor thrust line. The horizontal stabilizer/rudder unit comprises two half ellipses, with a major axis of $14 \mathrm{~cm}$, and minor axes of 4.763 and $2.223 \mathrm{~cm}$, respectively. Approximately $2.58 \mathrm{~cm}^{2}$ of the vertical stabilizer is occluded by the fuselage. By locating the rudder below the center-of-gravity (CG) of the craft, rudder deflection produces a sympathetic roll, i.e. a roll motion in the direction of the desired turn.

\begin{tabular}{|l|l|}
\hline Component & Specifications \\
\hline Aerial propulsion motor & Feigao Brushless DC motor $ø 13 \mathrm{~mm}, 36$ turn windings \\
\hline Electronic speed controller & Castle Creations Phoenix 10 Sensorless ESC \\
\hline Propeller & GWS EP3030 (ø=76mm) \\
\hline Control surface servos & Saturn S44 Digital Servo \\
\hline Terrestrial drive motors & Maxx Products MX-50HP \\
\hline Power storage & Polyquest 11.1V 600mAh Lithium-polymer Battery \\
\hline
\end{tabular}

Table II. List of critical components

\begin{tabular}{|lc|}
\hline Parameter & Value \\
\hline Wingspan (b) & $30.5 \mathrm{~cm}$ \\
\hline Wing area (S) & $364.4 \mathrm{~cm}^{2}$ \\
\hline Aspect Ratio (AR) & 2.55 \\
\hline
\end{tabular}

Table III. Main wing data

\begin{tabular}{|lc|}
\hline Element & Area \\
\hline Horizontal stabilizer & $52.3 \mathrm{~cm}^{2}$ \\
\hline Elevator & $24.4 \mathrm{~cm}^{2}$ \\
\hline Vertical stabilizer & $52.9 \mathrm{~cm}^{2}$ \\
\hline Rudder & $5.0 \mathrm{~cm}^{2}$ \\
\hline
\end{tabular}

Table IV. Control surface data 


\section{B. Multi-mode Locomotion}

Table V summarizes the aerial and terrestrial locomotion characteristics of MALV II. The maximum flight time was determined experimentally (flying the plane until the battery could not produce necessary thrust). Maximum crawling time was estimated by experimentally determining the current draw for locomotion on flat terrain, and comparing this to the battery capacity. However, its ground mobility is not restricted to flat terrain. It can crawl over grassy areas and climb obstacles higher than its leg length (Fig. 9).

It is apparent from the table that MALV possesses strong aerial range for its size, and is capable of considerable terrestrial locomotion within the capacity of a single battery pack. All control of MALV II is executed using standard R/C equipment. The transmitter's programmability facilitates fluid transition from flight to crawling control. During flight, the right joystick controls elevator (up/down, channel 1) and rudder (left/right, channel 2). Once the vehicle is landed, the operator switches the controller into ground mode, which then “mixes” the right joystick commands and transmits the results on channels 4 and 5. "Forward” (joystick up) sends positive signals to both channels, while "right” sends a positive signal to the left wheel-leg, and a negative signal to the right wheel-leg for differential steering. "Backward” and "left” act conversely. Channels 1 and 2 are turned off during ground mode.

\begin{tabular}{|l|c|}
\hline Parameter & Value \\
\hline Cruising air speed & $11 \mathrm{~m} / \mathrm{s}$ \\
\hline Maximum flight time & $15 \mathrm{~min}$ \\
\hline Flight range (round trip) & $4.9 \mathrm{~km}$ \\
\hline Maximum terrestrial speed & $0.33 \mathrm{~m} / \mathrm{s}$ \\
\hline Maximum crawling time & $100 \mathrm{~min}$ \\
\hline Terrestrial range (round trip) & $0.99 \mathrm{~km}$ \\
\hline
\end{tabular}

Table V. Nominal performance characteristics of the Micro Air Land Vehicle II

\section{Transition Between Flight and Crawling}

Beyond the capacity to both fly and crawl, perhaps the most challenging aspect of MALV design was to enable effective transition between the two locomotion modalities. MALV II is presently capable of transitioning from flying to crawling locomotion and, in some circumstances, capable of attaining flight from a crawling mode.

\section{1) Air-to-Land Transition}

Fig. 9 shows a snapshot sequence taken from a video of MALV flying, landing, and subsequently crawling (in this case to search for an object hidden in a road construction barrier). The dynamic mechanical properties of 
the vehicle absorb the energy of landing impact and enable effective and immediate crawling locomotion.

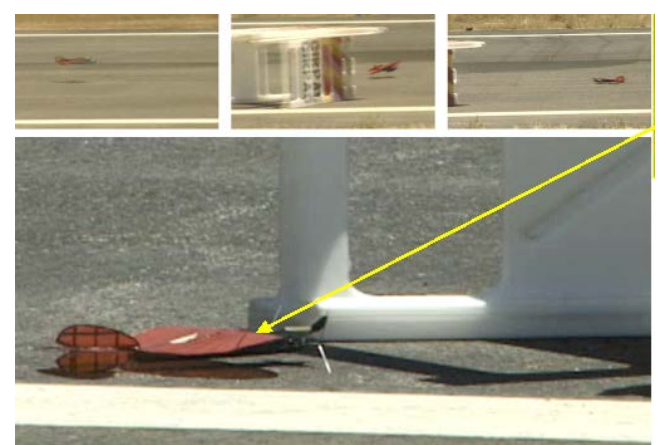

Fig 9. Snapshots of MALV II transitioning from flight to crawling locomotion

2) Land-to-Air Transition

The vehicle's ability to perform sufficiently at high angle-of-attack and low airspeed conditions resulted in a repeatable, successful take-off capability from atop building structures two stories or taller. After the vehicle walks off of the roof of the structure, it enters a powered dive, pulled down by both gravity and the propeller. As airspeed builds, the necessary lift is generated to arrest the fall and transition to flight phase. Take-off from a sloped roof-top produces more consistent results than from a cantilevered plate. The vehicle is able to attain a higher ground speed on the declined runway, thereby maintaining more favorable vehicle orientation at lift-off and gaining consistent separation from the building. Fig. 10 shows this sequence. MALV has also been shown to takeoff from the ground on hard, smooth surfaces such as concrete and asphalt using its propeller thrust. The wheel legs act like skids as the vehicle accelerates to take-off speed in 3 to 5 meters.

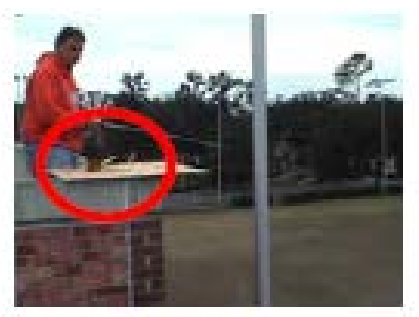

(a)

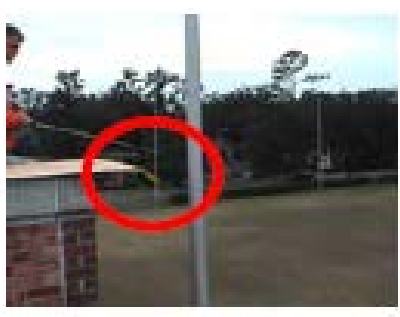

(b)

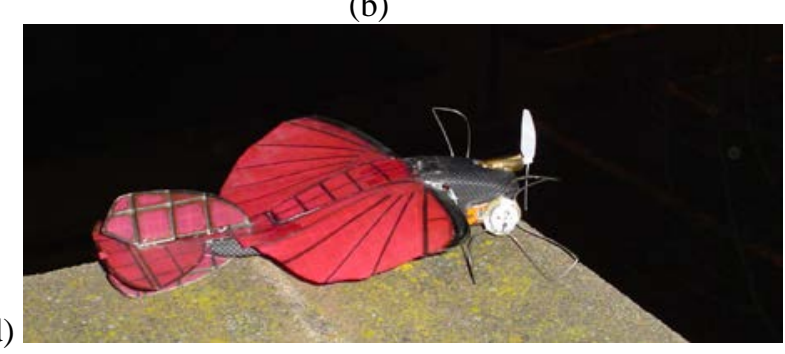

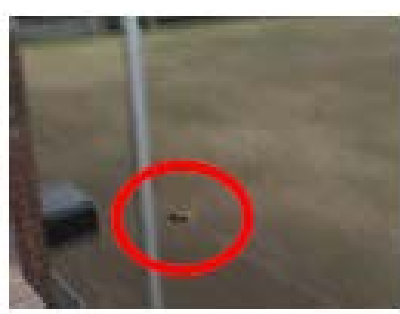

(c)

Fig. 10: a) MALV nears the edge of the structure; b) MALV walks off of the building, entering a "power dive"; c) lift is produced, and the vehicle pulls out of the dive; d) closeup of MALV prepared to walk off of a building.

\section{Sensor Capability and Integration}

MALV II originally used a 7.4V, 600mAh lithium polymer (Li-Poly) battery. With this power source, its 
Feigao propeller motor was able to produce a cruising speed of $8 \mathrm{~m} / \mathrm{s}(17.9 \mathrm{mph})$. The lift generated at this airspeed was nearly consumed by the weight of the terrestrial drive system and one camera/transmitter unit. Installing an $11.1 \mathrm{~V}, 600 \mathrm{mAh}$ battery increased the cruising speed to $11 \mathrm{~m} / \mathrm{s}$. The increased lift supported the increased battery mass (59g vs. 41g), a second camera, an electronic switch to control camera signal transmission, and the added mass of a 6 channel receiver. The camera transmitter operates in the $2.4 \mathrm{GHz}$ range. Fig. 12 shows an image capture of the transmission during a normal flight. Future work is envisaged for visionbased vehicle navigation based on the camera feedback.

\section{CONCLUSIONS}

This paper introduces a unique small ( 30cm maximum dimension) micro air-land vehicle (MALV) drawing from locomotion principles found in legged and winged animals. Much of the success of MALV is due to two biologically inspired mechanisms integrated into its design: a compliant wheel-leg terrestrial running gear and chord-wise compliant wings. Its wheel-legs mimic leg motions while rotating continuously and enable it to climb over terrestrial obstacles that are taller than its legs. MALV survives hard landings on concrete because its flexible wheel-legs passively comply during impact reducing the magnitude of the force transmitted to its onboard components. This mimics the same function of passive compliance found in the legs of an animal when it is suddenly perturbed. Likewise, MALV's chord-wise compliant wing overcomes many of the stability difficulties associated with flight on the micro air vehicle scale through a mechanism observed in animal flight, passive adaptive washout, wherein the shape of the wing passively adapts to variations in airflow. The MALV is human portable, hand launched, and radio controlled. It can fly several kilometers, land, and then crawl for many meters around the landing site while surmounting tall obstacles relative to its height. MALV transmits video signals from its position in the air or on the ground back to the pilot. When it lands on a building or other location that is at least two stories high, it can walk off of the structure and retake to the air. Furthermore, it can takeoff from the ground on hard, smooth surfaces. To our knowledge MALV is the first successful vehicle at this small scale to be capable of both flight and terrestrial locomotion in real-world terrains, and smooth transition between the two ${ }^{2}$. We believe it may provide a basis for design comparison for a range of future micro robots as research in this field matures.

Targeted applications include a wide range of search and rescue, safety, and security mission scenarios [30],[31]. Rescue, fire or police units would benefit from a small robotic vehicle easily transported and deployed

${ }^{2}$ Video of and from the robot during field testing may be found at: http://faculty.nps.edu/ravi/ 
by the unit to provide situational awareness in specific areas. Another application of a vehicle capable of flight and ground movement would be in detection of dangerous or illegal substances. While a mono-modal unmanned aerial vehicle (UAV) might be capable of identifying the existence of potential threats, closer inspection is required to evaluate the validity of the threat. A small vehicle with the ability to land near and walk up to the potential hiding location would allow the operator to accurately determine the present or absence of harmful or dangerous substances. A scalable family of vehicles with multiple modes of locomotion is envisioned in future work for all these applications.

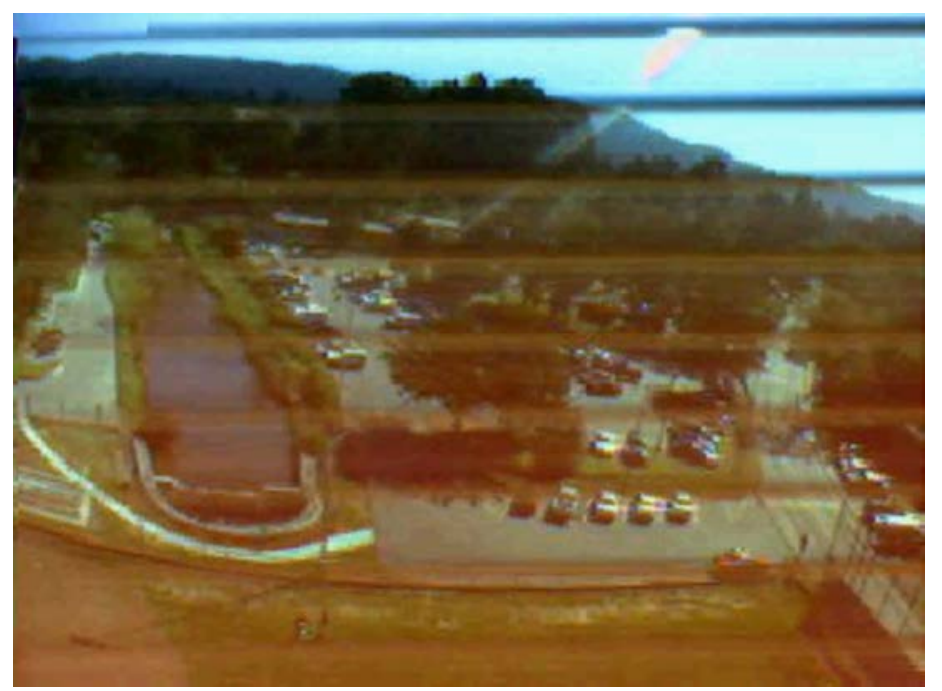

Fig. 11. Roads and cars are clearly visible in this image captured from video transmitted from MALV II in flight.

\section{ACKNOWLEDGMENTS}

The authors would like to acknowledge program support directors Dr. David Netzer of the Naval Postgraduate School (NPS)/USSCOM Cooperative Field Experimentation Program, and Chris Perry and Jeffery Wagner at US Air Force Research Laboratories Munitions Research Directorate for technical and mission planning insights. Dr. Kevin Jones provided assistance in sensor placement, and piloting/performance research. Baron Johnson and Daniel Claxton made significant contributions including vehicle design and flight-testing. Michael Sytsma, Michael Morton, and the University of Florida MAV group also contributed to the development, testing and analysis of MALV II.

\section{REFERENCES}

[1] P.G. Ifju, S. Ettinger, D.A. Jenkins, Y. Lian, W. Shyy, M.R. Waszak (2002), "Flexible-Wing-Based Micro Air Vehicles”, 40th AIAA Aerospace Sciences Meeting, Reno, NV AIAA 2002-0705, Jan. 2002.

[2] B.G.A. Lambrecht, A.D. Horchler, J.M. Morrey, R.E. Ritzmann, R.D. Quinn, (2004) "A Series of Highly Mobile and Robust Small Quadruped Robots,” Robotics and Autonomous Systems Journal, In Press.

[3] K-TEAM SA HEADQUARTERS SWITZERLAND Chemin du Vuasset, CP 111, 1028 Préverenges, SWITZERLAND. 
[4] Bererton C., L.E. Navarro-Serment, R. Grabowski, C.J.J. Paredis, P. K. Khosla, (2000), “Millibots: Small Distributed Robots for Surveillance and Mapping”. Government Microcircuit Applications Conference, 2023 March 2000.

[5] Fukui, R., A. Torii, A. Ueda, (2001), "Micro robot actuated by rapid deformation of piezoelectric elements,” Proceedings, 2001 International Symposium on Micromechatronics and Human Science, (MHS 2001), pp 117-22.

[6] Birch, M.C., R.D. Quinn, R.E. Ritzmann, A.J. Pollack, S.M Philips, (2002). "Micro-robots inspired by crickets.” Proceedings of Climbing and Walking Robots Conference (CLAWAR’02), Paris, France.

[7] Clark, J.E., Cham, J.G., Bailey, S.A., Froehlich, E.M., Nahata, P.K., Full, R.J., Cutkosky, M.R., Biomimetic design and fabrication of a hexapedal running robot, 2001. Proceedings 2001 ICRA. IEEE International Conference on Robotics and Automation, Vol.4, 2001, Pages: 3643- 3649 vol.4.

[8] Quinn, R.D., G.M. Nelson, R.E. Ritzmann, R.J. Bachmann, D.A. Kingsley, J.T. Offi, T.J. Allen, (2003), "Parallel Strategies For Implementing Biological Principles Into Mobile Robots," International Journal of Robotics Research, Vol. 22 (3) pp. 169-186.

[9] Ellington, C.P., “The Aerodynamics of Hovering Flight,” Philosophical Transactions of the Royal Society of London, Vol 305, No. 1122, 1984, pp. 1 181.

[10] Frampton, K. D., Goldfarb, M., Monopoli, D., \& Cveticanin, D., “Passive Aeroelastic Tailoring for Optimal Flapping Wings,” Proceeding of the Fixed, Flapping and Rotary Wing Vehicles at Very Low Reynolds Numbers, pp. 26 33, 2000.

[11] Jones, K.D., Duggan, S.J. \& Platzer, M.F., “Flapping-Wing Propulsion for a Micro Air Vehicle,” AIAA Paper No. 2001 0126, 2001.

[12] Jones, K.D., Bradshaw, C.J., Papadopoulos, J., and Platzer, M.F., "Improved Performance and Control of Flapping-Wing Propelled Micro Air Vehicles,” 42nd AIAA Aerospace Sciences Meeting and Exhibit, AIAA Paper 2004-0399, Reno, Nevada, January 5-8, 2004.

[13] Grasmeyer, J.M. \& Keennon, M.T., “Development of the Black Widow Micro Air Vehicle,” AIAA Paper No. 2001-0127, 2001.

[14] Morris, S., Holden, M., “Design of Micro Air Vehicles and Flight Test Validation,” Proceeding of the Fixed, Flapping and Rotary Wing Vehicles at Very Low Reynolds Numbers, pp.153 176, 2000.

[15] Frontiers of Engineering: Reports on Leading Edge Engineering, 2001 NAE Symposium on Frontiers of Engineering, National Academy of Engineering, pg. 12, 2002.

[16] Waszak, M. R., Jenkins, L. N., \& Ifju, P. G., “Stability and Control Properties of an Aeroelastic Fixed Wing Micro Aerial Vehicle,” AIAA 2001 4005.

[17] Shyy, W., Computational Modeling for Fluid Flow and Interfacial Transport, Elsevier, Amsterdam, The Netherlands, (1994, revised printing 1997).

[18] Shyy, W., Berg, M., Ljungqvist, D., "Flapping and Flexible Wings for Biological and Micro Vehicles," Process in Aerospace Sciences, Vol. 35, No. 5, 1999, pp. 455 506.

[19] Shyy, W., Thakur, S.S., Ouyang, H., Liu, J. \& Blosch, E., Computational Techniques for Complex Transport Phenomena, Cambridge University Press, New York, 1997.

[20] Shyy, W., Udaykumar, H. S., Rao, M.M \& Smith, R. W., Computational Fluid Dynamics with Moving Boundaries, Taylor \& Francis, Washington, DC, (1996, revised printing 1997 \& 1998).

[21] Smith, R. W., \& Shyy, W., "Computational Model of Flexible Membrane Wings in Steady Laminar Flow," AIAA Journal, Vol. 33, No. 10, 1995, pp. 1769 77.

[22] Jenkins D. A., Shyy, W., Sloan, J., Klevebring, F., \& Nilsson, M., “Airfoil Performance at Low Reynolds Numbers for Micro Air Vehicle Applications," Thirteenth Bristol International RPV/UAV Conference, University of Bristol, 1998.

[23] Ifju, P.G., Ettinger, S., Jenkins, D.A., \& Martinez, L., “Composite Materials for Micro Air Vehicles,” Proceeding for the SAMPE Annual Conference, Long Beach CA, May 6-10, 2001.

[24] Jenkins, D.A., Ifju, P.G., Abdulrahim, M., \& Olipra, S., “Assessment of the Controllability of Micro Air Vehicles,” Micro Air Vehicle Conference, Bristol England, April 2001.

[25] Ettinger, S.M., Nechyba, M.C., Ifju, P.G., \& Waszak, M., "Vision-Guided Flight Stability and Control for Micro Air Vehicles,” Proc. IEEE Int. Conf. on Intelligent Robots and Systems, vol. 3, pp. 2134 40, 2002.

[26] Lachat, D., A. Crespi, A.J. Ijspeert (2006), "Boxybot: A Swimming and Crawling Fish Robot Controlled by a Central Pattern Generator," Proceedings of The First IEEE / RAS-EMBS International Conference on Biomedical Robotics and Biomechatronics (BioRob 2006).

[27] Georgiadis, C., A. German, A. Hogue, H. Liu, C. Prahacs, A. Ripsman, R. Sim, L.-A. Torres, P. Zhang, M. Buehler, G. Dudek, M. Jenkin, E. Milios, (2004). "AQUA: An Aquatic Walking Robot." Proceedings of IEEE/RSJ International Conference on Intelligent Robots and Systems, IROS 2004, Sendai, Japan, September 28 - October 2, 2004. 
[28] Ayers, J., J.L. Davis, A. Rudolph, eds. (2002), Neurotechnology for Biomimetic Robots, The MIT Press, pp 481-509.

[29] Michelson, R., D. Helmick, S. Reece, C. Amareno (1997), “A Reciprocating Chemical Muscle (RCM) for Micro Air Vehicle "Entomopter" Flight,” AUVSI’97, Proceedings of the Association for Unmanned Vehicle Systems International, July 1997.

[30] Bachmann, R.J., F.J. Boria, P.G. Ifju, R.D. Quinn, J.E. Kline, R. Vaidyanathan (2005), “Utility of a Sensor Platform Capable of Aerial and Terrestrial Locomotion,” Proceedings of the IEEE/ASME International Conference on Advanced Intelligent Mechatronics (AIM2005), Monterey California, July, 2005.

[31]Boria F.J., R.J. Bachmann, P.G. Ifju, R.D. Quinn, R. Vaidyanathan, C. Perry, J. Wagener, “A Sensor Platform Capable of Aerial and Terrestrial Locomotion,” Proceedings of IEEE/RSJ 2005 International Conference on Intelligent Robots and Systems (IROS2005), Edmonton, Alberta, Canada, 2-6 August 2005.

[32] Kim, S., Clark, J.E., and Cutkosky, M.R. "iSprawl: Design and Tuning for High Speed Autonomous Open Loop Running,” International Journal of Robotics Research, V.25, No. 9, 2006.

[33]B. G. A. Lambrecht, A. D. Horchler, and R. D. Quinn, "A Small Insect Inspired Robot that Runs and Jumps," Proceeding of the IEEE International Conference on Robotics and Automation (ICRA '05), Barcelona, Spain, 2005.

[34] Watson, J.T., R.E. Ritzmann, S.N. Zill, A.J. Pollack (2002), "Control of obstacle climbing in the cockroach, Blaberus discoidalis: I. Kinematics,” Journal of Comparative Physiology, Vol. 188, p 39-53.

[35] T.J. Allen, R.D. Quinn, R.J. Bachmann, R.E. Ritzmann, “Abstracted Biological Principles Applied with Reduced Actuation Improve Mobility of Legged Vehicles,” Proceedings of IEEE International Conference on Intelligent Robots and Systems (IROS ’03), V.2, pp. 1370-1375, Las Vegas, USA.

[36] Saranli, U., Buehler, M. and Koditschek, D. (2001). RHex a simple and highly mobile hexapod robot. Int. J. Robotics Research, 20(7): 616-631.

[37] Morrey, J.M., Horchler, A.D., Didona, N., Lambrecht, B., Ritzmann, R.E. and Quinn, R.D. (submitted) Increasing Small Robot Mobility Via Abstracted Biological Inspiration, 2003 IEEE International Conference on Robotics and Automation (ICRA’03) Video Proceedings, Taiwan.

[38] Mueller, T. J. editor, "Proceedings of the Conference on Fixed, Flapping and Rotary Wing Vehicles at Very Low Reynolds Numbers,” Notre Dame University, Indiana, June 5-7, 2000.

[39] Mueller, T.J., “The Influence of Laminar Separation and Transition on Low Reynold's Number Airfoil Hysteresis,” J. Aircraft 22, pp. 763-770, 1985.

[40]Alexander, R.McN. "Three Uses for Springs in Legged Locomotion," International J. Robotics Research 9:2, 1990.

[41]Loeb GE, Brown IE and Cheng EJ (1999) A hierarchical foundation for models of sensorimotor control. Exp. Brain Res. 126: 1-18.

[42] Jindrich, D. L. and Full R.J. (2002). Dynamic stabilization of rapid hexapedal locomotion. J. Exp. Biol. 205: 2803-2823.

[43] Morasso, P. Bottaro, A., Casadio M., Vittorio Sanguineti "Preflexes and internal models in biomimetic robot systems”, Cogn Process, 6: 25-36, 2005

[44] Brown IE, Loeb GE, (1997) “A reductionist approach to creating and using neuromusculoskeletal models”, In: Biomechanics and neural control of movement, Winters JM, Crago PE (eds) Springer, Berlin Heidelberg New York, 148-163, 1997

[45] D. Lentink, N. Bradshaw, S.R. Jongerius (2007), “Novel micro aircraft inspired by insect flight”, abstract: A7.16, Comparative Biochemistry and Physiology, Part A 146 (2007) pages S133-S134. (http://www.delfly.nl/?site=DII\&menu=\&lang=en)

[46] Pringle, J.W.S. (1957) Insect Flight. Cambridge University Press, Cambridge.

[47] Ritzmann, RE, Fourtner, CR and Pollack, AJ (1983) Morphological and physiological identification of motor neurons innervating flight musculature in the cockroach, Periplaneta americana. J. Exp. Zool. 225:347-356.

[48] A. Ijspeert, A. Crespi, D. Ryczko, and J.-M. Cabelguen (2007), "From swimming to walking with a salamander robot driven by a spinal cord model”, Science, 315(5817):1416-1420, 2007. 